\title{
Hydro mechanical numerical modelling of geotechnical problems using local second gradient models
}

\author{
René Chambon ${ }^{1} \&$ Frédéric Collin ${ }^{2}$ \\ ${ }^{1}$ Laboratoire $3 S$, UJF - INPG - CNRS, Grenoble, France \\ ${ }^{2}$ FNRS Post-doctoral Researcher, ULg - Geomac, Belgium
}

The paper presents some applications of a local second gradient theory used to describe the mechanical behavior of a continua. This theory has been developed in order to avoid some well known drawbacks of classical theories, when softening and/or localization occur in a computation. This theory is extended and then applied in a hydromechanical coupled problem, in the case of fully saturated geomaterials. The corresponding finite element method taking into account the geometrical non linearities is detailed. Preliminary results using the finite element code developed in this context are then discussed and allow to study post localization behavior.

\section{INTRODUCTION}

This paper is devoted to some preliminary results concerning the use of a local second gradient model in initial boundary value problems in the context of hydromechanical coupled applications. This is mainly a work in progress. We focus on the equations solved and the finite element method used to tackle properly the problem.

Soils and rocks are mainly porous materials filled by a fluid. It is very important then to study numerically hydromechanical problems. This has been extensively done in the past and this paper is not intended neither to give new results within this field nor to discuss general equations (general means here taking into account unsaturated conditions). The reader interested by numerical computations of multiphasic materials can read for instance the book of Lewis and Schrefler (Lewis and Schrefler 2000). An other important reference dealing with hyper elasto plastic modelling is the paper written by Borja and Alarcon (Borja and Alarcon 1995).

When submitted to large strains, geomaterials exhibit clear localized ruptures, this phenomenon has been extensively studied, especially when the geomaterial is assumed to be a monophasic material. In this case, it is well known that it is necessary to use an enhanced model, in order to properly compute post localized paths. Many enhancements have been proposed and studied in the past. It is not the aim of this paper to compare the advantages and the shortcomings of every class of enhanced models. Readers interested by such a topic can have a look to the references of our paper coauthored with D. Caillerie and C. Tamagnini (Chambon, Caillerie, and Tamagnini 2004).

The present paper paper is restricted to the use of a local second gradient model in hydromechanical coupled problems. To our knowledge very few works have been done using an enhanced model in such problems. It is our opinion that the more important works done in this topic has been performed by Ehlers and coworkers. Since years they use Cosserat continuum in coupled problems within a large strain elasto plastic framework (Ehlers and Volk 1998) (Ehlers, Ellsiepen, and Ammann 2001). More recently some work has been carried out by Zhang and Shrefler (Zhang and Schrefler 2004) using a non local elasto plastic second gradient model.

Let us first give the main assumptions of this work. We are dealing with quasi static problems (which means that all inertia terms are neglected). The porous skeleton is assumed to be filled of fluid. The geometrical effects are taken into account (which means that for the skeleton the 
current configuration is different from the reference one). We are working within an elasto plastic framework, but the constitutive equation of the skeleton is an hypo elasto plastic model. It is not a hyper elasto plastic model belonging to the framework defined in (Chambon, Caillerie, and Tamagnini 2004).

The sequence of presentation is as follows. After a section giving the main notations, the third part is devoted to a presentation of the coupled classical (i.e. without second gradient effects) equations. Then a section deals with the enhancements induced by the use of a local second gradient model for the mechanical behavior of the skeleton. The fifth part is devoted to the finite element treatment of the problem. In the sixth section, examples of computations are detailed.

\section{NOTATIONS}

The main notations used in this paper are given here, some others are defined when they are used the first time. A component is denoted by the name of the tensor (or vector) accompanied with tensorial indices. All tensorial indices are in lower position as there is no need in the following of a distinction between covariant and contravariant components. The summation convention with respect to repeated tensorial indices is adopted throughout.

The time $t$ plays an important role in the following it is always indicated as an index in the upper position. Derivation with respect to time is denoted by a dot. These time derivative are material time derivatives, this means that they are computed for the same material (skeleton) mass.

All the equations are written in the current (or spatial) configuration of the skeleton at a given time $t$. All the functions defined in the following can be seen as functions of the reference position of the skeleton denoted $x_{i}^{0}$. Since the current configuration is known, the mappings $x_{i}^{t}\left(x_{i}^{0}\right)$ and $x_{i}^{0}\left(x_{i}^{t}\right)$ between this configuration and the reference one are known. Finally all the functions can be seen as function of $x_{i}^{0}$ or $x_{i}^{t}$. In order to simplify the notations we use the same notation for the functions and theirs values. So we can write for a given value $a$ : $a\left(x_{i}^{0}\right)=a\left(x_{i}^{0}\left(x_{i}^{t}\right)\right)=a\left(x_{i}^{t}\right)$.

Stresses are defined following the classical continuum mechanics conventions. Consequently positive pressures are in fact negative isotropic stresses.

- $\sigma_{i j}^{t}$ is the Cauchy stress of the mixture at the current time $t$,

- $u_{i}^{\star}$ is the kinematically admissible virtual displacement field,

- $\epsilon_{i j}^{\star}=\frac{1}{2}\left(\frac{\partial u_{i}}{\partial x_{j}^{t}}+\frac{\partial u_{j}}{\partial x_{i}^{t}}\right)$ is the kinematically admissible virtual displacement field,

- $\phi^{t}$ is the porosity defined as $\phi^{t}=\frac{\Omega^{w, t}}{\Omega^{t}}$ where $\Omega^{t}$ is the current volume of a given mass of skeleton and $\Omega^{w, t}$ the corresponding volume of water,

- $\varrho^{s}$ is the solid grain density,

- $\varrho^{w, t}$ is the fluid density,

- $g_{i}$ is the gravity acceleration,

- $g$ is the norm of $g_{i}$,

- $n_{j}^{t}$ is the normal to the boundary of the current configuration,

- $p^{t}$ is the pore pressure,

- $k^{w}$ is the fluid compressibility,

- $V_{i}^{W / S, t}$ is the true fluid velocity with respect to the solid phase,

- $\sigma_{i j}^{\prime t}$ is the effective stress tensor as defined by Terzaghi : $\sigma_{i j}^{t}=\sigma_{i j}^{\prime t}-p^{t} \delta_{i j}$,

- $\sigma_{m}^{\prime t}$ is the mean effective stress $\sigma_{m}^{\prime t}=\frac{\sigma_{i i}^{\prime t}}{3}$,

- $e$ is the mean strain,

- $\omega_{i j}$ is the spin tensor. 


\section{CLASSICAL COUPLED PROBLEMS}

The details of the following developments can be found in (Collin 2003). It is assumed that there is two immiscible phases: the porous skeleton and the fluid (water in the usual case). Phase changes like dissolution are not considered. Moreover solid grains are assumed to be incompressible.

\subsection{Balance equations}

The balance equations can be written separately for both phases or alternatively for the whole mixture and one of the two phases. Mass balance equations and momentum balance ones are written in the current solid configuration denoted $\Omega^{t}$.

\subsubsection{Balance of momentum}

These balance equations are established for the mixture and for the fluid phase. In the mixture momentum balance equation, there is then no interaction force between fluid phase and grain skeleton.

Balance of momentum for the mixture In a weak form (virtual work principle), we have:

$$
\int_{\Omega^{t}} \sigma_{i j}^{t} \epsilon_{i j}^{\star} d \Omega^{t}=\int_{\Omega^{t}}\left(\varrho^{s}\left(1-\phi^{t}\right)+\varrho^{w, t} \phi^{t}\right) g_{i} u_{i}^{\star} d \Omega^{t}+\int_{\Gamma_{\sigma}^{t}} \bar{t}_{i}^{t} u_{i}^{\star} d \Gamma^{t} .
$$

$\Gamma_{\sigma}^{t}$ is the part of the boundary where traction $\bar{t}_{i}^{t}$ are known. It is then necessary to prescribe the following boundary conditions for any point belonging to $\Gamma_{\sigma}^{t}$ :

$$
\sigma_{i j}^{t} n_{j}^{t}=\bar{t}_{i}^{t} .
$$

Balance of momentum for the fluid phase Fluid phase equation is written for a unit current volume of the mixture:

$$
\frac{\partial p^{t}}{\partial x_{i}^{t}}+F_{i}^{S / W, t}+\varrho^{w, t} g_{i}=0,
$$

where $\phi^{t}$ has been cancelled. $\phi^{t} F_{i}^{S / W, t}$ is in fact the true drag force applied on to the fluid.

\subsubsection{Mass balance equations}

Mass balance equations are written on one hand for the fluid and on the other hand for the solid phase.

Mass balance equation for the solid phase Since the current configuration used is the current configuration of the skeleton, the mass balance equation of the solid grains is necessarily met. For a given mixture volume $\Omega^{t}$, mass equation reads:

$$
\frac{\partial\left(\rho_{s}\left(1-\phi^{t}\right) \Omega^{t}\right)}{\partial t}=0 .
$$

Mass balance equation for the fluid In a weak form, this equation reads:

$$
\int_{\Omega^{t}}\left(\dot{M}^{t} p^{\star}-m_{i}^{t} \frac{\partial p^{\star}}{\partial x_{i}^{t}}\right) d \Omega^{t}=\int_{\Omega^{t}} Q^{t} p^{\star} d \Omega^{t}-\int_{\Gamma_{q}^{t}} \bar{q}^{t} p^{\star} d \Gamma^{t} .
$$

$Q^{t}$ is a sink term and $\Gamma_{q}^{t}$ is the part of the boundary where the input fluid mass per unit area $\bar{q}^{t}$ is prescribed:

$$
\bar{q}^{t}=m_{i}^{t} n_{i}^{t}
$$


The mass flow $m_{i}^{t}$ is defined as follows:

$$
m_{i}^{t}=\varrho^{w, t} \phi^{t} V_{i}^{W / S, t} .
$$

$V_{i}^{W / S, t}$ is the true fluid velocity in the current configuration. The so called Darcy velocity is given by $\phi^{t} V_{i}^{W / S, t}$.

For the volume $\Omega^{t}$, the fluid mass is equal to:

$$
M^{t}=\varrho^{w, t} \phi^{t} \Omega^{t} .
$$

\subsection{Constitutive equations}

Except for the non linear behavior of the skeleton, the constitutive equations are detailed in this section.

\subsubsection{Fluid behavior}

A compressible fluid is considered and the bulk modulus $k^{w}$ of the fluid is introduced. Variation of fluid density related to pressure changes is given by the following relationship:

$$
\dot{\varrho}^{w, t}=\frac{\varrho^{w, t}}{k^{w}} \dot{p}^{t} .
$$

\subsubsection{Viscous drag forces}

Viscous drag forces between the skeleton and the fluid are related to the Darcy velocity:

$$
F_{i}^{S / W, t}=\frac{\varrho^{w, t} \phi^{t} g}{K} V_{i}^{W / S, t}
$$

$F_{i}^{S / W, t}$ is the true drag force applied on the fluid by the skeleton for a current unit volume of the mixture divided by the porosity. $K$ is the Darcy permeability $(\mathrm{m} / \mathrm{s})$.

\subsubsection{Mixture behavior}

As we assume that the porous medium remains saturated and that the grains are incompressible, the Terzaghi's definition of effective stress holds:

$$
\sigma_{i j}^{t}=\sigma_{i j}^{\prime t}-p^{t} \delta_{i j}
$$

\subsection{Governing equations}

Finally governing equations are the balance equations (1) and (5) written in the current configuration, they hold for any time $t$ :

$$
\begin{aligned}
& \int_{\Omega^{t}} \sigma_{i j}^{t} \epsilon_{i j}^{\star} d \Omega^{t}=\int_{\Omega^{t}}\left(\varrho^{s}\left(1-\phi^{t}\right)+\varrho^{w, t} \phi^{t}\right) g_{i} u_{i}^{\star} d \Omega^{t}+\int_{\Gamma_{\sigma}^{t}} \bar{t}_{i}^{t} u_{i}^{\star} d \Gamma^{t}, \\
& \int_{\Omega^{t}}\left(\dot{M}^{t} p^{\star}-m_{i}^{t} \frac{\partial p^{\star}}{\partial x_{i}^{t}}\right) d \Omega^{t}=\int_{\Omega^{t}} Q^{t} p^{\star} d \Omega^{t}-\int_{\Gamma_{q}^{t}} \bar{q}^{t} p^{\star} d \Gamma^{t} .
\end{aligned}
$$

In equation (12), the total stress tensor $\sigma_{i j}^{t}$ is computed using equation (11) and the constitutive equation of the skeleton.

In the fluid mass balance equation, mass flow is given by equation (7). Substituting $F_{i}^{S / W, t}$ given by equation (10) in equation (3) yields $\phi^{t} V_{i}^{W / S, t}$. Then the fluid mass flow is rewritten as follows:

$$
m_{i}^{t}=-\frac{K}{g}\left(\frac{\partial p^{t}}{\partial x_{i}^{t}}+\varrho^{w, t} g_{i}\right)=-\varrho^{w, t} \frac{\kappa}{\mu}\left(\frac{\partial p^{t}}{\partial x_{i}^{t}}+\varrho^{w, t} g_{i}\right),
$$

where $\kappa$ is the intrinsic permeability and $\mu$ is the fluid viscosity. 
The fluid mass $M^{t}$ is defined in equation (8). The time derivative of this quantity is obtained directly by using equations (9) and (4) this yields:

$$
\dot{M}^{t}=\varrho^{w, t}\left[\frac{\dot{p}^{t}}{k^{w}} \phi^{t}+\frac{\dot{\Omega}^{t}}{\Omega^{t}}\right] \Omega^{t}
$$

\subsection{Linearized equations}

Similarly to the work of Borja (Borja and Alarcon 1995), we chose in this paper to get the linearized equation for the continuum problem instead of for the discretized one as it is more usually done. More details on the computation can be seen in a forthcoming paper (Collin, Chambon and Charlier 2004). We use as usual a step by step procedure to solve the initial boundary value problems.

Using a full Newton Raphson method for a given step yields the following linearized equations. A first guess of the solutions gives the so called out of balance forces $F_{i}^{\mathrm{HE}}$ and $F_{p}^{\mathrm{HE}}$ :

$$
\begin{array}{r}
\int_{\Omega^{\tau 1}} \sigma_{i j}^{\tau 1} \frac{\partial u_{i}^{\star}}{\partial x_{j}^{\tau 1}} d \Omega^{\tau 1}-\int_{\Omega^{\tau 1}}\left(\varrho^{s, \tau 1}\left(1-\phi^{\tau 1}\right)+\varrho^{w, \tau 1} \phi^{\tau 1}\right) g_{i} u_{i}^{\star} d \Omega^{\tau 1} \\
-\int_{\Gamma_{\sigma}^{\tau 1}} \bar{t}_{i}^{\tau 1} u_{i}^{\star} d \Gamma^{\tau 1}=\int_{\Omega^{\tau 1}} F_{i}^{\mathrm{HE}} u_{i}^{\star} d \Gamma^{\tau 1},
\end{array}
$$

and

$$
\begin{array}{r}
\int_{\Omega^{\tau 1}}\left(\dot{M^{\tau 1}} p^{\star}-m_{i}^{\tau 1} \frac{\partial p^{\star}}{\partial x_{i}^{\tau 1}}\right) d \Omega^{\tau 1}-\int_{\Omega^{\tau 1}} Q^{\tau 1} p^{\star} d \Omega^{\tau 1} \\
+\int_{\Gamma_{q}^{\tau 1}} \bar{q}^{\tau 1} p^{\star} d \Gamma^{\tau 1}=\int_{\Omega^{\tau 1}} F_{p}^{\mathrm{HE}} p^{\star} d \Gamma^{\tau 1} .
\end{array}
$$

The aim is now to find the displacement field $d u_{i}$ and $d p$ to be added to their respective current values to define a new current configuration $\Omega^{t}$ well balanced. If we assume that the skeleton behavior is governed by the Terzaghi effective stresses, we get for the balance of momentum linearized equations:

$$
\begin{array}{r}
\int_{\Omega^{t}} \frac{\partial u_{i}^{\star}}{\partial x_{k}^{t}}\left(d \sigma_{i k}^{\prime}-\sigma_{i j}^{t} \frac{\partial d u_{k}}{\partial x_{j}^{t}}+\sigma_{i k}^{t} \frac{\partial d u_{l}}{\partial x_{l}^{t}}\right) d \Omega^{t}-\int_{\Omega^{t}}\left[\varrho^{s, t}\left(1-\phi^{t}\right) \frac{\partial d u_{l}}{\partial x_{l}^{t}}\right. \\
\left.-\varrho^{s, t}\left(1-\phi^{t}\right) \frac{d \Omega^{t}}{\Omega^{t}}+\varrho^{w, t}\left(1-\phi^{t}\right) \frac{d \Omega^{t}}{\Omega^{t}}+\varrho^{w, t} \phi^{t} \frac{\partial d u_{l}}{\partial x_{l}^{t}}\right] g_{i} u_{i}^{\star} d \Omega^{t} \\
+\int_{\Omega^{t}} \frac{\partial u_{i}^{\star}}{\partial x_{k}^{t}}\left(-d p^{t} \delta_{i k}\right) d \Omega^{t}-\int_{\Omega^{t}}\left(\varrho^{w, t} \frac{d p^{t}}{k^{w}} \phi^{t}\right) g_{i} u_{i}^{\star} d \Omega^{t}=\int_{\Omega^{t}} F_{i}^{\mathrm{HE}} u_{i}^{\star} d \Gamma^{t}
\end{array}
$$

The left hand side of equation (18) has two main contributions : the first one (corresponding to the first two terms) is related to classical expression taking into account geometrical non linear effects. The second contribution (last two terms) is related to the couplings between pore pressure and the balance of momentum equation.

For the fluid the mass conservation equation, the following linearized expression is obtained:

$$
\int_{\Omega^{t}}\left(\varrho^{w, t} \frac{d p^{t}}{k^{w}} \frac{\phi^{t}}{k^{w}} \dot{p}^{t}+\varrho^{w, t} \frac{\phi^{t}}{k^{w}} \frac{d p^{t}}{d t}+\varrho^{w, t} \frac{d p^{t}}{k^{w}} \frac{\dot{\Omega}^{t}}{\Omega^{t}}\right) p^{\star} d \Omega^{t}-\int_{\Omega^{t}}\left(-\varrho^{w, t} \frac{d p^{t}}{k^{w}} \frac{\kappa}{\mu}\right.
$$




$$
\begin{array}{r}
\left.\left(\frac{\partial p^{t}}{\partial x_{k}^{t}}+\varrho^{w, t} g_{k}\right)-\varrho^{w, t} \frac{\kappa}{\mu}\left(\frac{\partial d p^{t}}{\partial x_{k}^{t}}+\varrho^{w, t} \frac{d p^{t}}{k^{w}} g_{k}\right)\right) \frac{\partial p^{\star}}{\partial x_{k}^{t}} d \Omega^{t}+ \\
\int_{\Omega^{t}}\left(\varrho^{w, t} \frac{1}{k^{w}}\left(1-\phi^{t}\right) \frac{d \Omega^{t}}{\Omega^{t}} \dot{p}^{t}+\varrho^{w, t}\left(\frac{d \Omega^{t}}{\Omega^{t} d t}-\frac{\dot{\Omega}^{t}}{\Omega^{t}} \frac{d \Omega^{t}}{\Omega^{t}}\right)\right. \\
\left.+\varrho^{w, t}\left(\frac{\phi^{t}}{k^{w}} \dot{p}^{t}+\frac{\dot{\Omega}^{t}}{\Omega^{t}}\right) \frac{\partial d u_{i}}{\partial x_{i}^{t}}\right) p^{\star} d \Omega^{t}-\int_{\Omega^{t}}\left(\varrho^{w, t} \frac{\kappa}{\mu} \frac{\partial d u_{i}}{\partial x_{k}^{t}} \frac{\partial p^{t}}{\partial x_{i}^{t}}\right. \\
\left.-\varrho^{w, t} \frac{\kappa}{\mu}\left(\frac{\partial p^{t}}{\partial x_{i}^{t}}+\varrho^{w, t} g_{i}\right) \frac{\partial d u_{k}}{\partial x_{i}^{t}}-\varrho^{w, t} \frac{\kappa}{\mu}\left(\frac{\partial p^{t}}{\partial x_{k}^{t}}+\varrho^{w, t} g_{k}\right) \frac{\partial d u_{i}}{\partial x_{i}^{t}}\right) \frac{\partial p^{\star}}{\partial x_{k}^{t}} d \Omega^{t} \\
=\int_{\Omega^{t}} F_{p}^{\mathrm{HE}} p^{\star} d \Gamma^{t} .
\end{array}
$$

In equation (19), the first two terms are related to the classical expression for a flow problem. The last terms are related to the influence of the mechanical model on the mass balance equation (including geometrical non linear effects).

\section{LOCAL SECOND GRADIENT COUPLED MODEL}

The proposed coupled model is an extension of local second gradient model developed by one of the authors (Chambon, Caillerie, and Matsushima 2001). This kind of models can be traced back to the pioneering work of Cosserat brothers (Cosserat and Cosserat 1909), Mindlind (Mindlin 1965) and Germain (Germain 1973). One of the advantages of local second gradient approach is the fact that constitutive equations are local and therefore it is straightforward to formulate a second gradient extension of any classical models.

\subsection{Monophasic medium}

In the framework of microstructure continuum theory, a micro kinematic gradient field $v_{i j}$ is introduced to describe strain and rotation at the level of the grains. Compare to classical continuum mechanics, additional terms are then added in the internal virtual work for a given body (Germain 1973):

$$
W_{i}^{*}=\int_{\Omega^{t}}\left(\sigma_{i j} \epsilon_{i j}^{*}+\tau_{i j}\left(v_{i j}^{*}-F_{i j}^{*}\right)+\Sigma_{i j k} h_{i j k}^{*}\right) d \Omega^{t},
$$

where $F_{i j}^{*}$ is the virtual macro displacement gradient, $v_{i j}^{*}$ is the virtual micro kinematic gradient, $\tau_{i j}$ called microstress is an additive stress associated to the microstructure, $h_{i j k}^{*}$ is the virtual micro second gradient and $\Sigma_{i j k}$ is the double stress dual of $h_{i j k}^{*}$.

In the model developed in the following, an assumption is added: the micro kinematic gradient is equal to the macro displacement gradient (and so $v_{i j}^{*}=F_{i j}^{*}$ ). Consequently, the principle of virtual work can be rewritten as follows:

$$
\int_{\Omega^{t}}\left(\sigma_{i j} \epsilon_{i j}^{*}+\Sigma_{i j k} \frac{\partial^{2} u_{i}^{*}}{\partial x_{j} \partial x_{k}}\right) d \Omega^{t}=W_{e}^{*}
$$

The previous virtual work equation of second gradient models can of course be used in a finite element code. However, this equation needs the use of $C^{1}$ functions for the displacement field as second derivatives of the displacement are involved. In order to avoid such functions, the equality of $v_{i j}^{*}$ and $F_{i j}^{*}$ is introduced through a field of Lagrange multipliers $\lambda_{i j}$ related to a weak form of the constraint (Chambon, Caillerie, and ElHassan 1998):

$$
\int_{\Omega^{t}}\left(\sigma_{i j} \frac{\partial u_{i}^{*}}{\partial x_{j}}+\Sigma_{i j k} \frac{\partial v_{i j}^{*}}{\partial x_{k}}\right) d \Omega^{t}-\int_{\Omega^{t}} \lambda_{i j}\left(\frac{\partial u_{i}^{*}}{\partial x_{j}}-v_{i j}^{*}\right) d \Omega^{t}-W_{e}^{*}=0
$$




$$
\int_{\Omega^{t}} \lambda_{i j}^{*}\left(\frac{\partial u_{i}}{\partial x_{j}}-v_{i j}\right) d \Omega^{t}=0
$$

where $W_{e}^{*}$ is the external virtual work defined as follows:

$$
W_{e}^{*}=\int_{\Omega^{t}} \rho g_{i} u_{i}^{*} d \Omega^{t}+\int_{\Gamma^{t}}\left(\bar{t}_{i} u_{i}^{*}+\bar{T}_{i} v_{i k}^{*} n_{k}\right) d \Gamma^{t}
$$

\subsection{Extension to biphasic medium}

Starting from equations (12) and (13) of the coupled problem in classical mechanics, one has now to introduce the microstructure effects. The main assumption is the following: pore fluid has no influence at the level of the microstructure. The governing equations of the local second gradient coupled problem are then the following:

$$
\begin{aligned}
& \int_{\Omega^{t}}\left(\sigma_{i j} \frac{\partial u_{i}^{*}}{\partial x_{j}}+\Sigma_{i j k} \frac{\partial v_{i j}^{*}}{\partial x_{k}}\right) d \Omega^{t}-\int_{\Omega^{t}} \lambda_{i j}\left(\frac{\partial u_{i}^{*}}{\partial x_{j}}-v_{i j}^{*}\right) d \Omega^{t}= \\
& \int_{\Omega^{t}}\left(\varrho^{s}(1-\phi)+\varrho^{w} \phi\right) g_{i} u_{i}^{*} d \Omega^{t}+\int_{\Gamma^{t}}\left(\bar{t}_{i} u_{i}^{*}+\bar{T}_{i} v_{i k}^{*} n_{k}\right) d \Gamma^{t} \\
& \int_{\Omega^{t}} \lambda_{i j}^{*}\left(\frac{\partial u_{i}}{\partial x_{j}}-v_{i j}\right) d \Omega^{t}=0 \\
& \int_{\Omega^{t}}\left(\dot{M}^{t} p^{\star}-m_{i}^{t} \frac{\partial p^{\star}}{\partial x_{i}^{t}}\right) d \Omega^{t}=\int_{\Omega^{t}} Q^{t} p^{\star} d \Omega^{t}-\int_{\Gamma_{q}^{t}} \bar{q}^{t} p^{\star} d \Gamma^{t},
\end{aligned}
$$

Equation (25) is the balance of momentum of a biphasic medium with microstructure. The stress tensor $\sigma_{i j}$ is a total stress defined according to Terzaghi's postulate, on the contrary the double stress $\Sigma_{i j k}$ does not depend on pore pressure.

\section{COUPLED FINITE ELEMENT FORMULATION}

Similarly to equations (12) and equation (13), equations (25), (26) and (27) are linearized which allow us to define fields of out of balance forces. Details will be found in (Collin, Chambon and Charlier 2004). Then the resulting equations are spatially discretized using isoparametric finite elements with eight nodes for $u_{i}$ and $p$, four nodes for $v_{i j}$ and one node for $\lambda_{i j}$ (Figure 1). The usual quadratic serendipity shape function are used for $u_{i}$ and $p$. The linear shape function are used for $v_{i j}$, and $\lambda_{i j}$ is assumed to be constant.

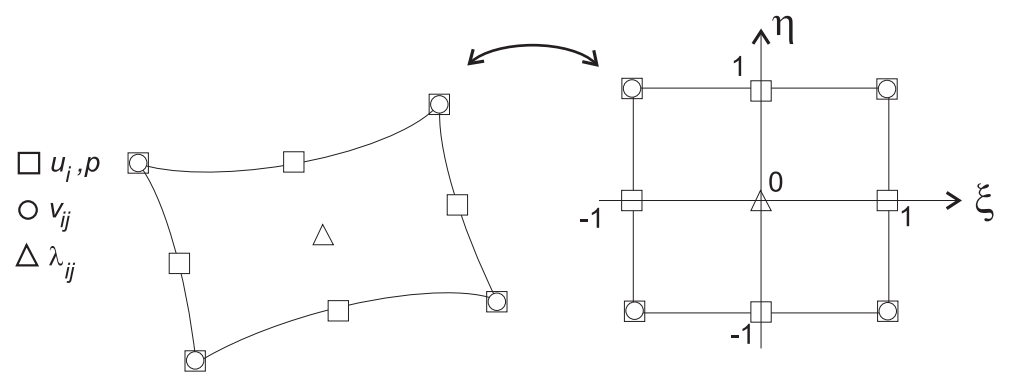

(a)

(b)

Figure 1: Quadrilateral element and parent element: (a) quadrilateral element; and (b) parent element 
As compare to monophasic local second gradient element, the definition of the elementary out of balance forces $\left[f_{H E}^{t}\right]$ and of the element stiffness matrix $\left[k^{t}\right]$ are the same (see (Matsushima, Chambon, and Caillerie 2002)):

$$
\begin{gathered}
{\left[U_{\text {node }}^{*}\right]\left[f_{H E}^{t}\right] \equiv W_{e}^{t^{*}}-\left[U_{\text {node }}^{*}\right]^{T} \int_{-1}^{1} \int_{-1}^{1}[B]^{T}\left[T^{t}\right]^{T}\left[\sigma^{t}\right] \cdot \operatorname{det} J^{t} d \xi d \eta} \\
{\left[U_{\text {node }}^{*}\right]^{T}\left[k^{t}\right]\left[d U_{\text {node }}^{t}\right]=} \\
{\left[U_{\text {node }}^{*}\right]^{T} \int_{-1}^{1} \int_{-1}^{1}[B]^{T}\left[T^{t}\right]^{T}\left[E^{t}\right]^{T}\left[T^{t}\right][B] \operatorname{det} J^{t} d \xi d \eta\left[d U_{\text {node }}^{t}\right]}
\end{gathered}
$$

But the transformation matrices $[B],\left[T^{t}\right]$, the stress vector $\left[\sigma^{t}\right]$ and the matrix $\left[E^{t}\right]$ have to be modified in order to introduce the terms relative to mass balance equation. For example, the definition of the matrix $\left[E^{t}\right]$ becomes:

$$
\left[E^{t}\right]=\left[\begin{array}{ccccc}
E 1_{(4 \times 4)}^{t} & K_{W M_{(4 \times 1)}} & 0_{(4 \times 8)} & 0_{(4 \times 4)} & -I_{(4 \times 4)} \\
K_{M W_{(1 \times 4)}} & K_{W W_{(1 \times 1)}} & 0_{(1 \times 8)} & 0_{(1 \times 4)} & 0_{(1 \times 4)} \\
E 2_{(8 \times 4)}^{t} & 0_{(8 \times 1)} & D_{(8 \times 8)}^{t} & 0_{(8 \times 4)} & 0_{(8 \times 4)} \\
E 3_{(4 \times 4)}^{t} & 0_{(4 \times 1)} & 0_{(4 \times 8)} & 0_{(4 \times 4)} & I_{(4 \times 4)} \\
E 4_{(4 \times 4)}^{t} & 0_{(4 \times 1)} & 0_{(4 \times 8)} & -I_{(4 \times 4)} & 0_{(4 \times 4)}
\end{array}\right]
$$

Matrices $K_{W W}, K_{W M}$ and $K_{M W}$ can be computed thanks to linearized equations (18) and (19). The full stiffness matrix is computed at each iteration of the Newtown-Raphson procedure.

\section{NUMERICAL APPLICATION OF LOCAL SECOND GRADIENT COUPLED MODEL}

Bi-axial experiments are known to exhibit clearly strain localization either in soil or rock samples (see for instance (Desrues 1984)). We have done a modelling of such a plane strain compression test in order to study the regularization of the problem in a bi-phasic medium. In this first approach, a very simple constitutive equation has been chosen. A classical model based on the Prandtl-Reuss elasto-plastic model gives the stress tensor $\sigma_{i j}$ as follows:

$$
\begin{aligned}
& \dot{\sigma}_{m}^{\prime}=3 K \dot{e} \\
& \tilde{\hat{\sigma}}_{i j}=\left\{\begin{array}{cl}
2 G_{1} \dot{\hat{\epsilon}}_{i j} & \left(\|\hat{\epsilon}\|<e_{l i m}\right) \\
2 G_{1}\left(\dot{\hat{\epsilon}}_{i j}-\frac{G_{1}-G_{2}}{G_{1}} \frac{\hat{\sigma}_{k l} \dot{\hat{\epsilon}}_{k l}}{\|\hat{\sigma}\|^{2}} \hat{\sigma}_{i j}\right) & \left(\|\hat{\epsilon}\|>e_{l i m}\right)
\end{array}\right.
\end{aligned}
$$

$K$ is the bulk modulus, $G_{1}$ and $G_{2}$ are the shear moduli before and after the peak respectively, $\dot{\sigma}_{m}$ is the mean stress rate, $\dot{e}$ is the mean strain rate, $\tilde{\hat{\sigma}}_{i j}$ is the Jaumann rate of the deviatoric Cauchy stress tensor and $\dot{\hat{\epsilon}}_{i j}$ are the deviatoric strain rates. $\|\hat{\epsilon}\|$ and $\|\hat{\sigma}\|$ are the second invariant of the deviatoric strain and stress respectively and $e_{l i m}$ is a model parameter.

An exponential function is assumed for the shear modulus $G_{2}$ after the yield point, so that the material could reach its residual state smoothly:

$$
G_{2}=\bar{G}_{2} \exp \left(\frac{\bar{G}_{2}}{G_{1} e_{\text {lim }}-\hat{\sigma}_{\text {res }}}\left(\|\hat{\epsilon}\|-e_{\text {lim }}\right)\right)
$$

$\bar{G}_{2}$ is the value of the shear modulus just after the peak and $\hat{\sigma}_{r e s}$ is the residual value of the second invariant of deviatoric stress tensor. 
For the second gradient part of the model, a simple isotropic linear relationship deduces from Mindling (Mindlin 1965) is used. It depends only on one parameter denoted $D$ :

$$
\left[\begin{array}{l}
\tilde{\Sigma}_{111} \\
\tilde{\Sigma}_{112} \\
\tilde{\Sigma}_{121} \\
\tilde{\Sigma}_{122} \\
\tilde{\Sigma}_{211} \\
\tilde{\Sigma}_{212} \\
\tilde{\Sigma}_{221} \\
\tilde{\Sigma}_{222}
\end{array}\right]=\left[\begin{array}{cccccccc}
D & 0 & 0 & 0 & 0 & \frac{D}{2} & \frac{D}{2} & 0 \\
0 & \frac{D}{2} & \frac{D}{2} & 0 & -\frac{D}{2} & 0 & 0 & \frac{D}{2} \\
0 & \frac{D}{2} & \frac{D}{2} & 0 & -\frac{D}{2} & 0 & 0 & \frac{D}{2} \\
0 & 0 & 0 & D & 0 & -\frac{D}{2} & -\frac{D}{2} & 0 \\
0 & -\frac{D}{2} & -\frac{D}{2} & 0 & D & 0 & 0 & 0 \\
\frac{D}{2} & 0 & 0 & -\frac{D}{2} & 0 & \frac{D}{2} & \frac{D}{2} & 0 \\
\frac{D}{2} & 0 & 0 & -\frac{D}{2} & 0 & \frac{D}{2} & \frac{D}{2} & 0 \\
0 & \frac{D}{2} & \frac{D}{2} & 0 & 0 & 0 & 0 & 0
\end{array}\right]\left[\begin{array}{l}
\frac{\partial \dot{v}_{11}}{\partial x_{1}} \\
\frac{\partial \dot{v}_{11}}{\partial x_{2}} \\
\frac{\partial v_{12}}{\partial x_{1}} \\
\frac{\partial v_{12}}{\partial x_{2}} \\
\frac{\partial v_{21}}{\partial x_{1}} \\
\frac{\partial \dot{v}_{21}}{\partial x_{2}} \\
\frac{\partial \dot{v}_{22}}{\partial x_{1}} \\
\frac{\partial \dot{v}_{22}}{\partial x_{2}}
\end{array}\right]
$$

$\dot{v}_{i j}$ is the material time derivative of $v_{i j}, \tilde{\Sigma}_{i j k}$ is the Jaumann double stress rate defined by the following equation:

$$
\tilde{\Sigma}_{i j k}=\dot{\Sigma}_{i j k}+\Sigma_{l j k} \omega_{l i}+\Sigma_{i m k} \omega_{m j}+\Sigma_{i j p} \omega_{p k},
$$

where $\omega_{l i}$ is the spin tensor.

A drained strain-controlled bi-axial test has been modelled. The loading strain rate is $0.18 \%$ per hour. The bottom plate is smooth, rigid and remains horizontal. The central point of this plate is horizontally fixed in order to avoid rigid body displacement. The top plate is also smooth and rigid, and a prescribed vertical displacement is then applied to every node of the mesh. No confining pressure is prescribed. The external additional double forces per unit area $P_{i}$ are assumed to by equal to zero all along the boundaries. The initial pore pressure is equal to zero. Pore pressures are fixed to their initial value at the top and bottom edges, in order to model the drainage system.

The parameters used for the simulations are listed in the Table 1. Two values of intrinsic permeability are given and allow to show the influence of that property on the localization pattern.

Table 1: Parameters of the models

\begin{tabular}{lll|lll|lll}
\hline \multicolumn{6}{l}{ First Gradient model } & \multicolumn{5}{c}{ Second Gradient model } & \multicolumn{1}{l}{ Flow model } \\
\hline$K$ & 97.3856 & $\mathrm{MPa}$ & $D$ & 500 & $\mathrm{~N}$ & $\kappa$ & $10^{-19} / 10^{-12}$ & $\mathrm{~m}^{2}$ \\
$G_{1}$ & 50 & $\mathrm{MPa}$ & & & $\rho_{w}$ & 1000 & $\mathrm{~kg} / \mathrm{m}^{3}$ \\
$\bar{G}_{2}$ & -2 & $\mathrm{MPa}$ & & & $\phi$ & 0.15 & - \\
$e_{\text {lim }}$ & 0.01 & - & & & $k_{w}$ & $510^{-10}$ & $\mathrm{~Pa}^{-1}$ \\
$\hat{\sigma}_{\text {res }}$ & $0.2 \sigma_{\text {peak }}$ & $\mathrm{MPa}$ & & & $\mu_{w}$ & 0.001 & $\mathrm{~Pa} . \mathrm{s}$ \\
\hline
\end{tabular}

In order to exhibit strain localization, a imperfection is introduced in the bottom left finite element. In this element, the parameter $e_{l i m}$ is $10 \%$ lower than the one used for the others.

For the first simulation, the intrinsic permeability is equal to $10^{-12} \mathrm{~m}^{2}$. For such values of loading rate and permeability, overpressures in the sample are very low and the corresponding loading curve is equivalent to the curve obtained with a monophasic local second gradient model (Figure 3). Figure 2 shows us the localization pattern clearly initiated by the material default and the water flows related to the drainage of the sample.

Other simulations of biaxial experiment have been performed with a permeability value of $10^{-19} \mathrm{~m}^{2}$. In fact, for such a value, the loading rate is so fast that the drainage is not possible and the curve corresponds to an undrained biaxial test. Different mesh sizes have been used and Figure 3 shows us that the corresponding load-displacement curves are more or less merged. Equivalent strains are plotted on Figure 4 for $20 \times 10,30 \times 15,40 \times 20$ elements meshes. One sees clearly the zone where strains are localized. Before the localization process, the strain in the sample is homogeneous. Just after localization, the geomaterial inside the shear band is plastic, but it remains elastic outside. This is quite clear in Figure 5: a square is plotted for each plastic Gauss point. This allows us to accurately measure the thickness of the shear band in our computations.

These preliminary computations show that the permeability has an influence on the loading curve (Figure 3) but not on the shear band thickness. This observation is of course only valid for the model we use in the modelling. 


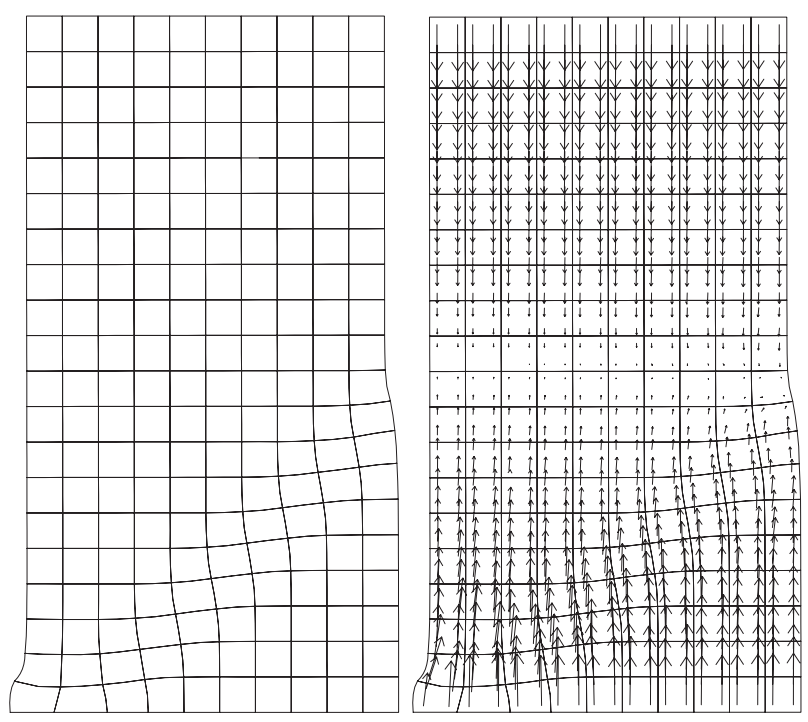

Figure 2: Localization mode and Water flow after $2.5 \%$ axial strain $\left(\kappa=10^{-12} \mathrm{~m}^{2}\right)$

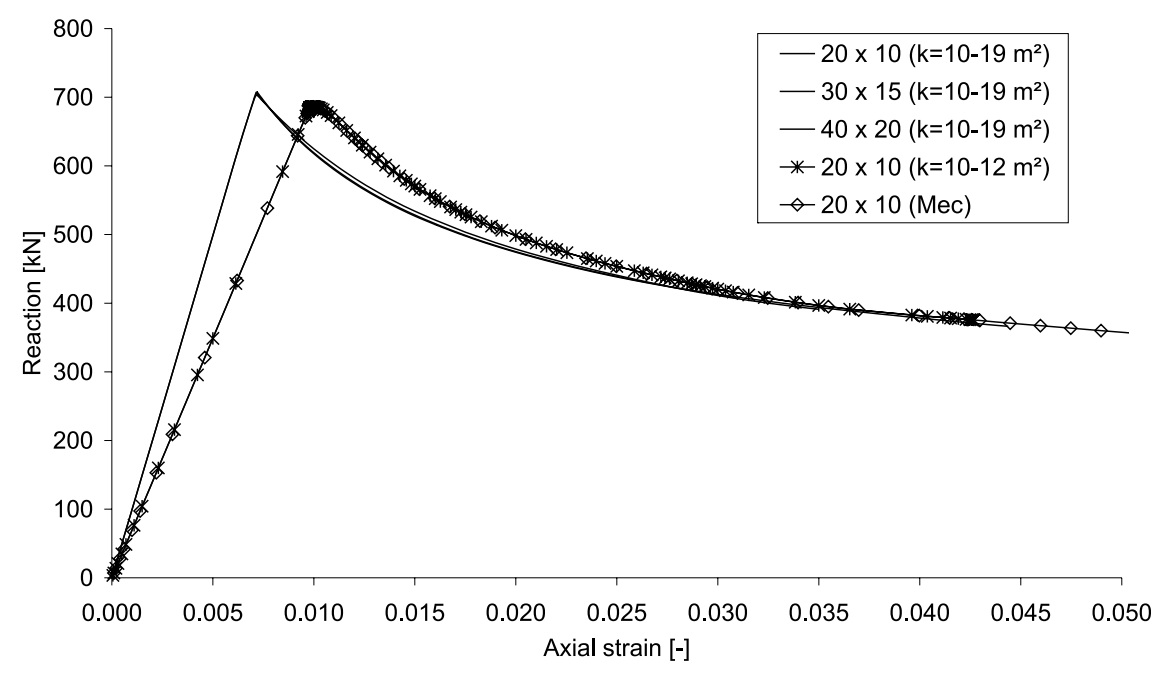

Figure 3: Axial strain vs Global reaction for 5 biaxial tests

\section{CONCLUSIONS}

Enhanced models are necessary to obtain regularized solutions for localized plastic strain fields computed with a finite element method. Many enhancements have been developed in the past, especially in the case of monophasic materials. However, geomaterials are mainly porous media filled by one or several fluids and it is thus interesting to study the influence of the fluid on the localization problem.

In this paper, our analysis is restricted to saturated soils or rocks filled by one compressible pore fluid. A coupled second gradient formulation has been proposed for this analysis. A finite element code using this local second gradient model has been presented and biaxial tests have been modelled.

Numerical results show clearly the regularization of the localization pattern. This study is only a preliminary one and some further investigations are necessary in order to have more clear conclusion. 

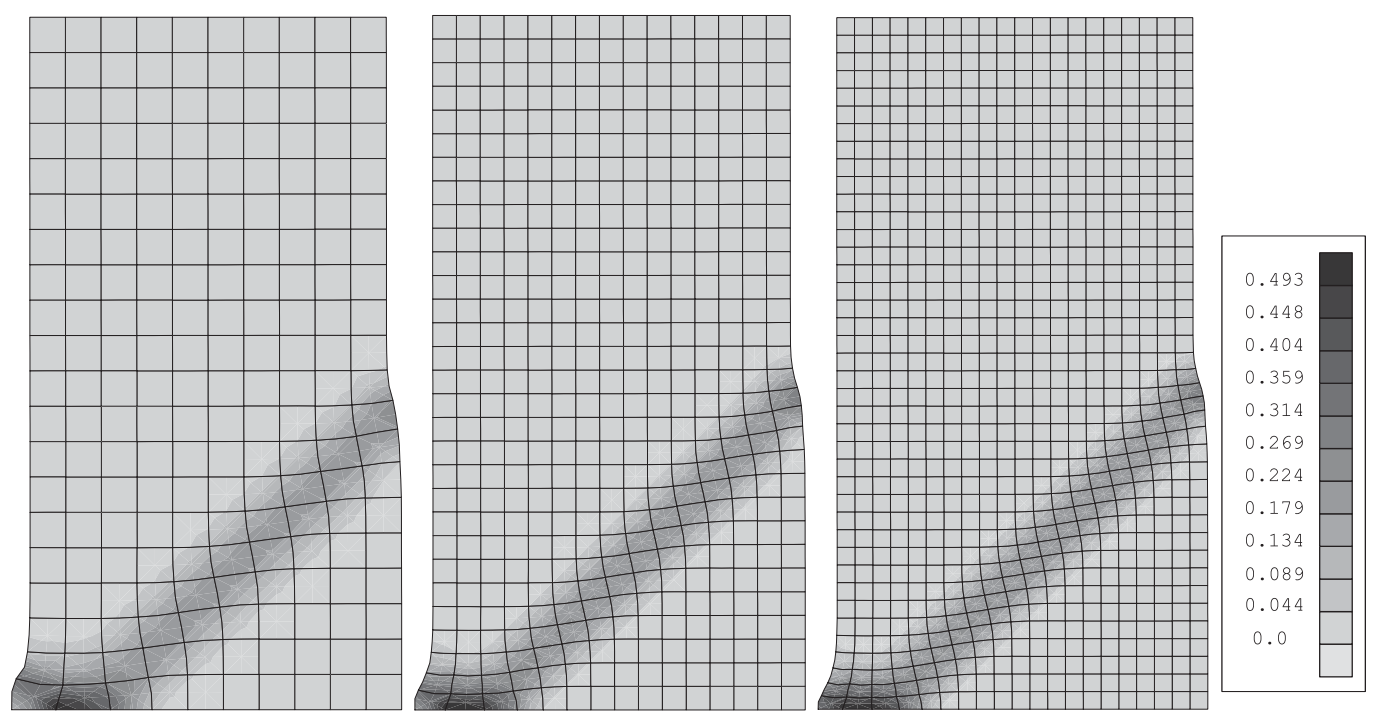

Figure 4: Deviatoric deformation after $2.5 \%$ axial strain $\left(\kappa=10^{-19} \mathrm{~m}^{2}\right)$
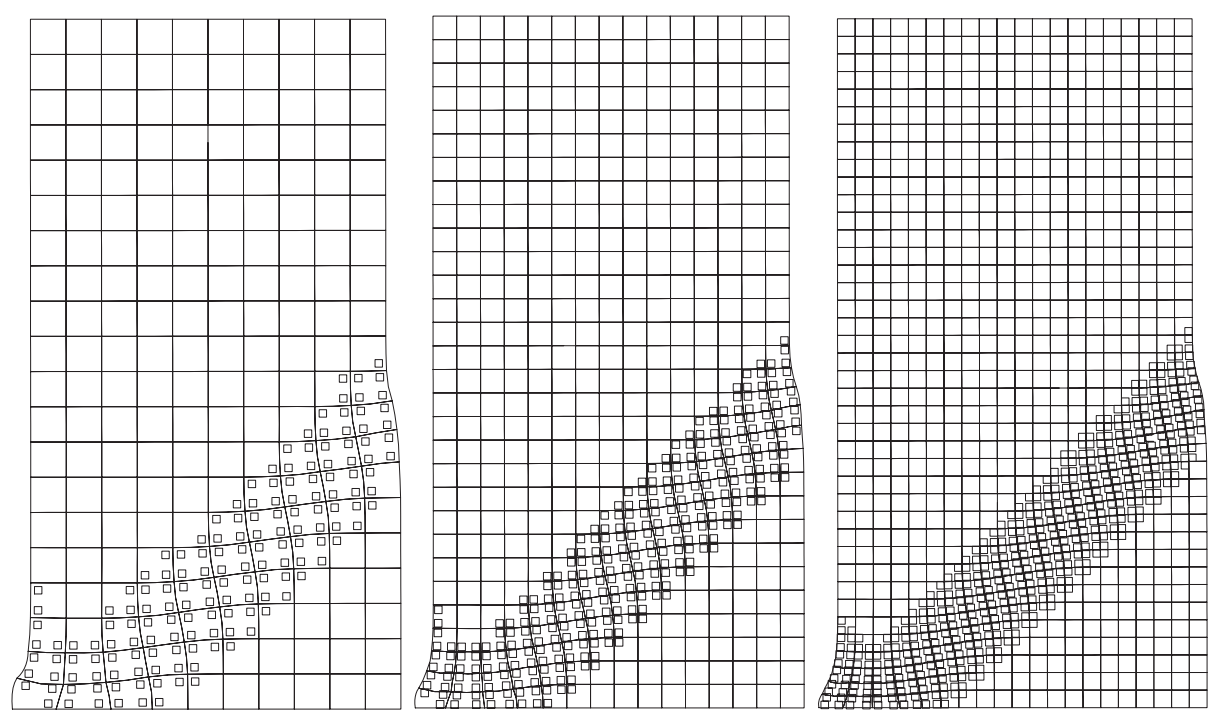

Figure 5: Plastic zones after $2.5 \%$ axial strain $\left(\kappa=10^{-19} \mathrm{~m}^{2}\right)$

Acknowledgement The results presented in this paper have been obtained during a first post doctoral stay in Laboratoires 3S-Grenoble with a financial support of the European project RTNDIGA (Contract No. HPRN-CT-2002-00220) and a second post doctoral stay granted by Fonds National de la Recherche Scientifique of Belgium. They are both gratefully acknowledge for their supports.

\section{REFERENCES}

Borja, R. and E. Alarcon (1995). A mathematical framework for finite strain elastoplastic consolidation part 1: Balance law, variational formulation and linearization. Computers methods in Applied Mechanics and Engineering 122, 145-171.

Chambon, R., D. Caillerie, and N. ElHassan (1998). One-dimensional localization studied with a second grade model. European Journal of Mechanics A/solids 17, 637-656.

Chambon, R., D. Caillerie, and T. Matsushima (2001). Plastic continuum with microstructure, local second gradient theories for geomaterials: localization studies. International Journal of Solids and Structures 38, 8503-8527.

Chambon, R., D. Caillerie, and C. Tamagnini (2004). A strain gradient plasticity theory for finite strain. Computer Methods in Applied Mechanics and Engineering 193, 2797-2826. 
Collin, F. (2003). Couplages thermo-hydro-mecaniques dans les sols et les roches tendres partiellement saturees. These de Doctorat, Universite de Liege (231), 300p.

Collin, F., R. Chambon and R. Charlier (2004). Computation of coupled problem with a local second gradient model. in preparation.

Cosserat, E. and F. Cosserat (1909). Theorie des corps deformables. A Hermann et Fils: Paris.

Desrues, J. (1984). La localisation de la deformation dans les materiaux granulaires. These de Doctorat es Sciences.

Ehlers, W., P. Ellsiepen, and M. Ammann (2001). Time- and space-adaptive methods applied to localization phenomena in empty and saturated micropolar and standard porous media. International Journal for numerical methods in engineering, 503-526.

Ehlers, W. and W. Volk (1998). On theoretical and numerical methods in the theory of porous media based on polar and non-polar elsto-plastic solid materials. International Journal of Solids and Strutures 35, 4597-4617.

Germain, P. (1973). La methode des puissances virtuelles en mecanique des milieux continus: Premiere partie : theorie du second gradient. Journal de mecanique 12(2), 235-274.

Lewis, R. and B. Schrefler (2000). The finite element method in the static and dynamic deformation and consolidation of porous media. J. Wiley \& Sons.

Matsushima, T., R. Chambon, and D. Caillerie (2002). Large strain finite element analysis of a local second gradient model: application to localization. International Journal for Numerical Methods in Engineering 54, 499-521.

Mindlin, R. (1965). Second gradient of strain and surface-tension in linear elasticity. Int. j.Solids Structures 1, $417-438$.

Zhang, C. and B. Schrefler (2004). Particular aspects of internal length scales in strain localization analysis of multiphase porous materials. Computer Methods in Applied Mechanics and Engineering 193, 2867-2884. 